\title{
Indirect 3D and 4D Printing of Soft Robotic Microstructures
}

\section{Journal Article}

\section{Author(s):}

De Marco, Carmela (D); Alcantara, Carlos; Kim, Sangwon; Briatico, Francesco; Kadioglu, Ahmet; de Bernardis, Gaston; Chen, Xiangzhong; Marano, Claudia; Nelson, Bradley (D); Pané, Salvador

\section{Publication date:}

2019-09

\section{Permanent link:}

https://doi.org/10.3929/ethz-b-000359536

Rights / license:

In Copyright - Non-Commercial Use Permitted

Originally published in:

Advanced Materials and Technologies 4(9), https://doi.org/10.1002/admt.201900332

Funding acknowledgement:

702128 - 3D-printed magnetic microfluidics for applications in life sciences (EC) 


\section{WILEY-VCH}

DOI: $10.1002 /(($ please add manuscript number $))$

\section{Article type: Communication}

\section{Indirect 3D and 4D Printing of Soft Robotic Microstructures}

Carmela De Marco*\&1${ }^{1}$, Carlos Alcantara\& ${ }^{1}$, Sangwon Kim ${ }^{1}$, Francesco Briatico ${ }^{3}$, Ahmet Kadioglu ${ }^{1}$, Gaston De Bernardis ${ }^{2}$, Xiangzhong Chen ${ }^{1}$, Claudia Marano ${ }^{3}$, Bradley J. Nelson ${ }^{1}$ and Salvador Pané ${ }^{1}$

${ }^{1}$ Multi-Scale Robotics Laboratory, Institute of Robotics and Intelligent Systems, ETH Zurich, Tannenstrasse 3, CH-8092 Zürich, Switzerland.

${ }^{2}$ Kantonnspital Aarau, Tellstrasse 25, CH-5001 Aarau, Switzerland

${ }^{3}$ Department of Chemistry, Materials and Chemical Engineering, Politecnico di Milano

*e-mail: demarcoc@ethz.ch

(\& These authors contributed equally)

Keywords: 3D printing, 4D printing, hydrogels, shape memory polymers, microstructures, soft robotics, direct laser writing

The development of 3D soft-robotic components is currently hindered by material limitations associated with conventional 3D printing techniques. To overcome this challenge, we propose an indirect $3 \mathrm{D}$ printing approach based on the fabrication of $3 \mathrm{D}$ printed sacrificial templates. High-resolution micromolds produced by direct laser writing were infused with polymers and then dissolved, leading to the final $3 \mathrm{D}$ printed soft microstructures. We used this method to indirectly print 3D and 4D soft-microrobots. The versatility of our technique is shown through the fabrication and actuation of gelatin helices filled with magnetic nanoparticles. In addition, we show that stent-like microstructures with shape memory properties can be manufactured with minimum features of $5 \mu \mathrm{m}$, which is 40 times smaller than those reported to date. In summary, the utilization of this technique can overcome obstacles associated with the fabrication of soft microrobots and surgical tools for minimally invasive surgery. 


\section{WILEY-VCH}

Untethered small-scale robots have emerged as promising tools for biomedical applications such as targeted drug and cell delivery, localized diagnosis, and biopsies ${ }^{[1-3]}$. Over the last decade, the field of small-scale robotics has explored how these small devices should swim ${ }^{[4,5]}$, adapt their shapes ${ }^{[6-8]}$, and controllably carry and release therapeutic cargos. ${ }^{[9-14]}$ While recent research efforts have also been made towards the use of these tiny machines in vivo ${ }^{[15-17]}$, several aspects need to be synergistically addressed in order to ultimately provide relevant clinical solutions with micro and nanorobotic technologies. As in the field of robotics ${ }^{[18]}$, a widespread tendency today among small-scale roboticists is to substitute hard components with softer elements, including parts made from elastomers, hydrogels, macromolecular systems and biomolecules. This paradigm shift in materials is a logical development from the locomotion and actuation point of view. Soft materials allow for more sophisticated movements such as deformation, shrinkage/swelling, and changes in morphology $[19,20]$. Additionally, soft materials display mechanical properties that are much closer to those of biological structures such as tissues, and they usually exhibit enhanced biocompatibility characteristics. Soft matter can also be programmed to biodegrade by the body's chemistry, for example, by enzymes or $\mathrm{pH}^{[9,10]}$. Consequently, the production of micro- and nanorobotic platforms from soft building blocks will advance the field of small-scale robotics (in terms of material constituents) towards medical applications.

An impediment to the miniaturization of small soft components lies in the available manufacturing methods and their related limitations, which are mainly a result of the physicochemical nature of the soft material. In order to fabricate micro- and nanodevices with superior capabilities, the production of components with any conceivable shape is essential. To date, 3D printing (3DP) techniques have offered a wealth of opportunities for creating 3D structures with virtually unlimited shapes or materials. 3DP has also strongly impacted the field of robotics, by enabling the fabrication of soft robotic engines with sophisticated 3D continuous movements. Although substantial research has been devoted to the production of 


\section{WILEY-VCH}

3D printed small-scale robotic tools, these are typically made of non-responsive, stiff materials ${ }^{[21-23]}$ or, in the case of soft materials, their geometrical features are either rudimentary, or too large (from $\sim 200 \mu \mathrm{m}$ to a few $\mathrm{mm}$ ) to be used as biomedical micro or nanorobots ${ }^{[24-26]}$.

Here, we propose a method that uses the capabilities of Direct Laser Writing (DLW ${ }^{[27]}$ to produce complex 3D sacrificial templates for molding polymers that cannot be directly 3D printed at the microscale by any other technique. One of the first examples of using sacrificial templates obtained by DLW showed the fabrication of gold helices as photonic metamaterials as broadband circular polarizer ${ }^{[28]}$. Electrodeposition in DLW templates has also been utilized for the fabrication of magnetic microrobots ${ }^{[29,30]}$. However, DLW templates have not yet been used for the 3D shaping of polymers at the microscale. The indirect 3D printing technique we propose enables the fabrication of $3 \mathrm{D}$ and $4 \mathrm{D}$ soft robotic structures at the microscale. The process is schematically depicted in Figure 1. We demonstrated the potential of this approach with two examples of microrobotic structures made from two different types of polymers: (a) gelatin, a non-photocurable hydrogel, with and without nanoparticles embedded in the matrix and (b) a mercapto-ester polyurethane-based shape-memory polymer (SMP). The indirect 3D printing method used to shape these two different polymers is schematically presented in Figure $1 \mathrm{~A}$ and Figure 1B, respectively. 


\section{WILEY-VCH}

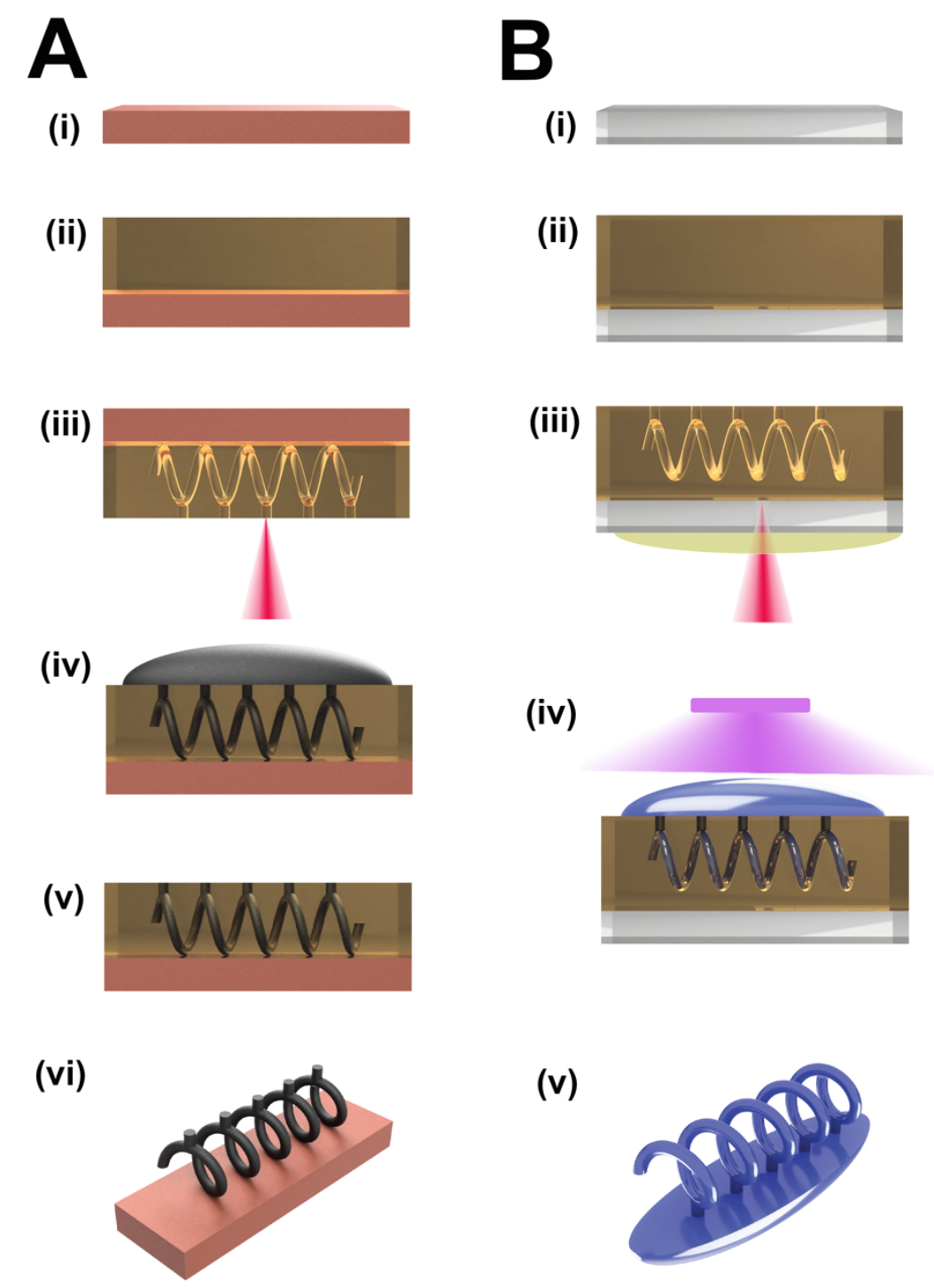

Figure 1 (A) Schematic of the indirect 3D-printing process: a silicon substrate (i) is spin-coated with a positivetone photoresist (ii) and the 3D template is written into the photoresist by DLW (iii), developed afterwards and filled with the hydrogel (iv). After gelification (v), the 3D printed microswimmers are obtained by stripping away the photoresist (vi). (B) Schematic of the indirect 4D-printing process: a glass slide (i) is spin-coated with a positive-tone photoresist (ii) and the 3D template is written into the photoresist by DLW (iii), developed afterwards and filled with the curable SMP (iii). After curing under UV light (iv), the 4D printed microstructure is obtained by stripping away the photoresist (v).

\section{Indirect $3 D$ printing of gelatin microstructures}

We have chosen to focus on gelatin as the hydrogel and helix as the shape for two reasons: gelatin is a hydrogel extensively used in tissue engineering and bioprinting for its high biocompatibility ${ }^{[31,32]}$, and helical microstructures, also known as artificial bacterial flagella (ABFs), are widely adopted microrobotic designs as they can propel by corkscrew locomotion with low rotating magnetic fields ${ }^{[33,34]}$. Although we have recently shown that biodegradable 


\section{WILEY-VCH}

gelatin-based ABFs can be directly fabricated by DLW ${ }^{[10]}$, gelatin has to be functionalized with acrylic groups in order to be photocurable. The addition of photosensitive moieties to polymers to enable their processing with photolithography is an interesting strategy. However, the introduction of functional groups such as acrylates significantly decreases their biocompatibility and hampers their use for biomedical applications ${ }^{[35]}$.

Here, for the first time, we have 3D shaped gelatin at the microscale without the need for additional functionalization. The indirect 3D printing process we used is shown in Figure 1A. We spin-coated a silicon substrate (Figure 1A(i)) with a positive-tone photoresist, followed by a soft-bake process (Figure 1A(ii)). The 3D shape was defined using a commercial DLW system (Figure 1A(iii)). After development, the template was filled with the gelatin solution (Figure 1A(iv)). A pure gelatin solution, obtained by mixing gelatin and distilled water in a weight ratio of 90:10, and a composite gelatin solution, obtained by adding a $5 \% \mathrm{w} / \mathrm{w}$ of magnetic nanoparticles (MNPs) to the previous solution, were investigated. The composite gelatin helices were magnetized perpendicularly on the long axis, under an external magnetic coil system with $20 \mathrm{mT}$ of field magnitude. The gelation of the helices was carried out overnight at $5^{\circ} \mathrm{C}$ (Figure $\left.1 \mathrm{~A}(\mathrm{v})\right)$. The sacrificial templates were removed by placing the structures in acetone for about 20 minutes, leading to the final gelatin helices (Figure 1A(vi)). The optical images of the fabricated gelatin helices, the composite helices and the composite helices swollen in water after 5 minutes at room temperature, are respectively shown in Figure 2(a), (b) and (c). We carried out swimming tests under a rotating magnetic field to investigate the locomotion capabilities of magnetic composite helices. Some frames captured from the relative video are shown in Figure 2(d). Figure S1 shows the forward velocity of the gelatin composite helices as a function of the applied rotating frequency of a magnetic field at $20 \mathrm{mT}$, and the magnetic properties of the gelatin composite. 


\section{WILEY-VCH}

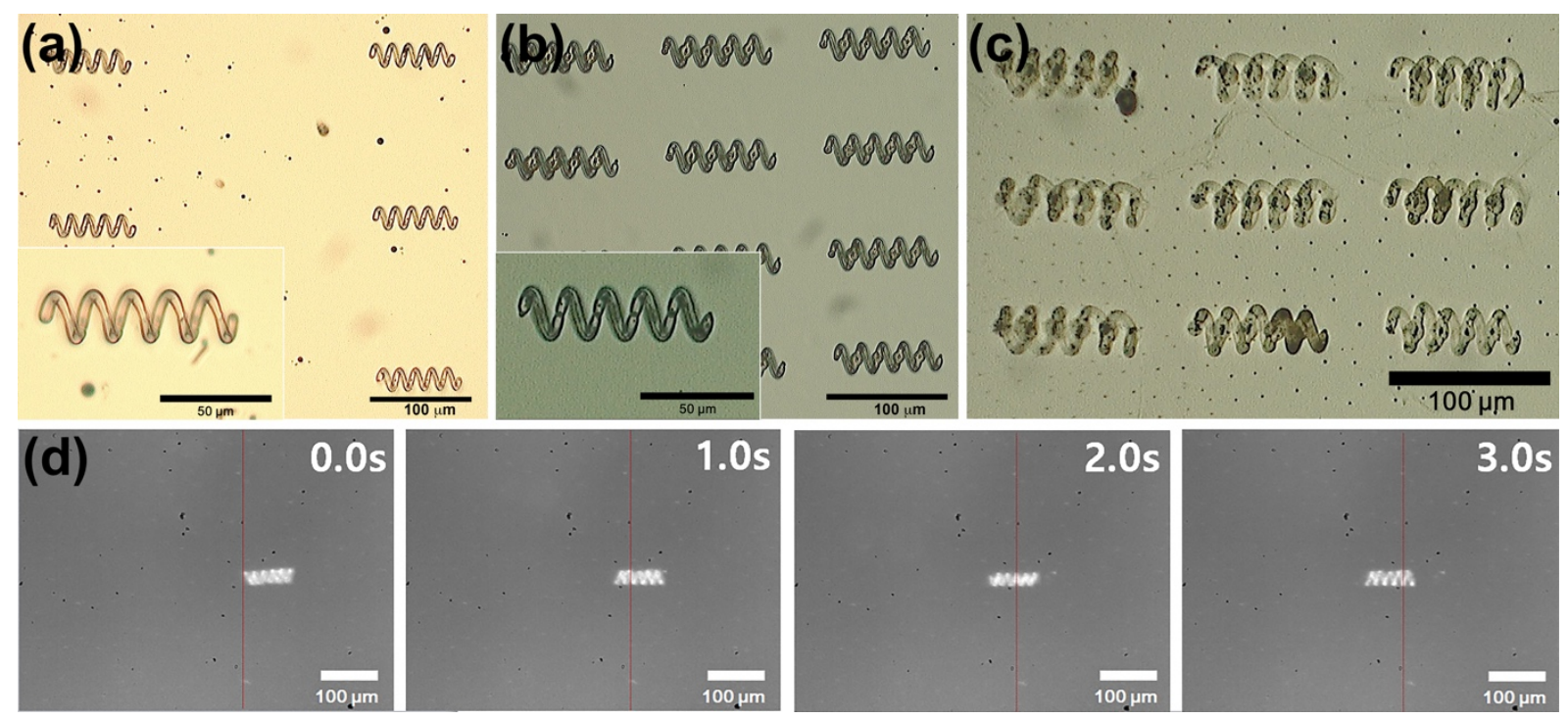

Figure 2 (a) Optical image of gelatin and (b) Gelatin magnetic composite helical microswimmers (magnification inset). (c) Gelatin composite helical microswimmers swollen in water. (d) Frames captured from a video showing the locomotion of gelatin composite helices under a rotating magnetic field.

\section{Indirect $4 D$ printing of SMP stent-like microstructures}

We recently highlighted how robotics could evolve using 3D printed smart materials ${ }^{[36]}$, that is, materials which undergo physical or chemical changes under the application of external stimuli. This concept led to the development of $4 \mathrm{D}$ printing ${ }^{[37]}$. To date, shape-memory polymers (SMPs) ${ }^{[38-40]}$ are the most investigated materials used in $4 \mathrm{D}$ printing. The use of 4D printing in the field of small-scale robots remains unexplored because of the challenges associated with miniaturizing smart materials using available 3D printing techniques. In fact, the few reported examples of 4D printing by Direct Ink Writing (DIW) have resolutions in the range from $\sim 200 \mu \mathrm{m}$ to a few millimetres ${ }^{[41-46]}$. However, DLW is a highly promising technique, because it enables the fabrication of high-resolution 3D microarchitectures with heights ranging from a few hundred nanometers up to several millimeters, and layer thicknesses below $1 \mu \mathrm{m}$. 3D printing of SMPs using DLW has not been yet reported. Finding the right composition of monomers and photoinitiators to obtain biocompatible SMPs, with operation temperatures in the range of body temperature, remains challenging, and is the 


\section{WILEY-VCH}

primary reason that SMPs have not been fabricated using DLW, even though this technique has been available for more than 20 years ${ }^{[47]}$.

To demonstrate the potential of indirect 4D printing in the field of soft robotics, we decided to extend our strategy to SMPs to show that this technique can also be used to 4D print soft microstructures. We selected NOA63 as an SMP because it is a commercially available UV optical adhesive. As a prototypical microstructure, we chose a stent-like shape due to its complex features. Note that no medical microstent at such a scale has been documented to date. Hence, this work could pave the way for future developments in medical conditions where microstents would be necessary. NOA63 material is often used in microfluidics because of its hydrophilicity, solvent resistance, optical transparency, and low cost. NOA glues are also biocompatible according to animal tests carried out by the supplier ${ }^{[48]}$. Although NOA63 is UV curable, DLW cannot be used to 3D print these complex structures directly, and the only reported usage with DLW refers to the fabrication of very simple $2.5 \mathrm{D}$ optical lenses on the top of an optical fiber ${ }^{[49]}$. The indirect 4D printing approach overcomes the limitations associated with 3D printing of SMPs at the microscale. Figure 1B shows the indirect 4D-printing process we carried out. We spin-coated a glass slide (Figure 1B(i)) with a positive-tone photoresist, followed by a soft-bake process lasting several minutes (Figure 1B(ii)). The 3D shape was defined using a commercial DLW system (Figure 1B(iii)). We chose two different stent-like geometries, one with acute-angle crowns, and one with an obtuse angles. 


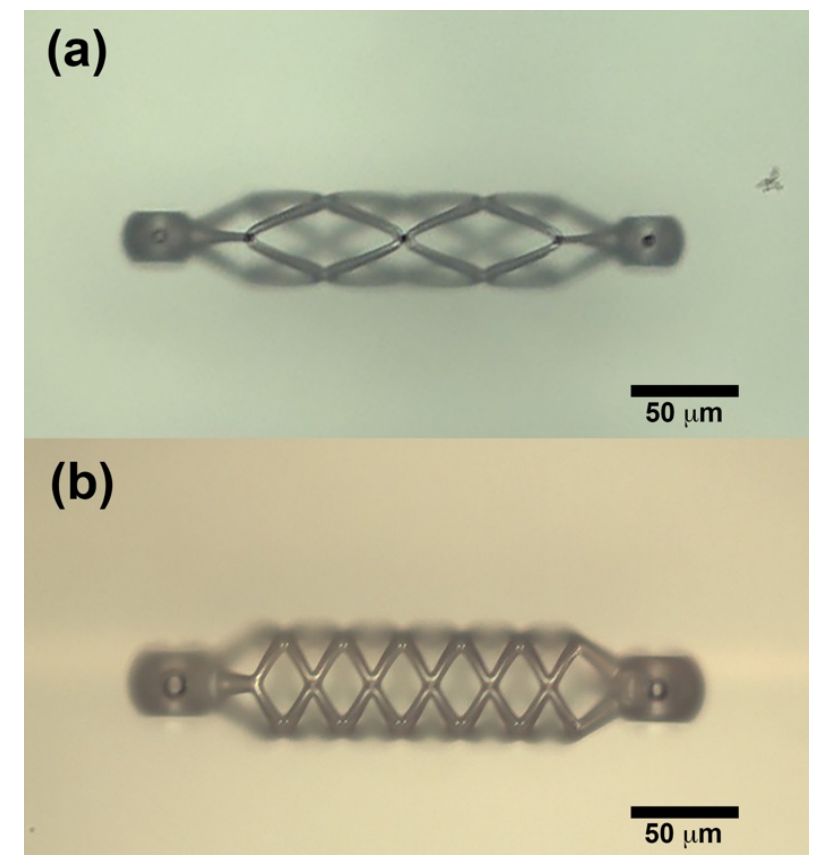

Figure 3 Empty sacrificial templates of stent-like shapes with an acute (a) and an obtuse (b) angle.

Figure 3(a) and (b) show the empty sacrificial templates after development. After fabricating the template, a few droplets of NOA63 were cast onto it and it was left to settle overnight. The template was then irradiated under UV light at $365 \mathrm{~nm}\left(2.7 \mathrm{~mW} / \mathrm{cm}^{2}\right)$ for 2 hours at room temperature (Figure 1B(iv)). Finally, the structure was placed in DMSO for 20 minutes to remove the template, it was then rinsed in water and dried with a nitrogen gun to achieve the desired 4D printed microstent (Figure 1B(v)).

The resulting indirect 4D printed microstructures were morphologically characterized by SEM (Figure 4). The microstructures were fabricated in an array (Figure 4(a)), with an acute angle (Figure 4(b) - magnification in Figure 4(c)) and an obtuse angle (Figure 4(d) magnification in Figure 4(e)). The microstents were designed with two lateral hooks so that they could be stretched during the mechanical test to assess their shape memory nature. A magnified SEM image is shown in Figure 4(f). 


\section{WILEY-VCH}
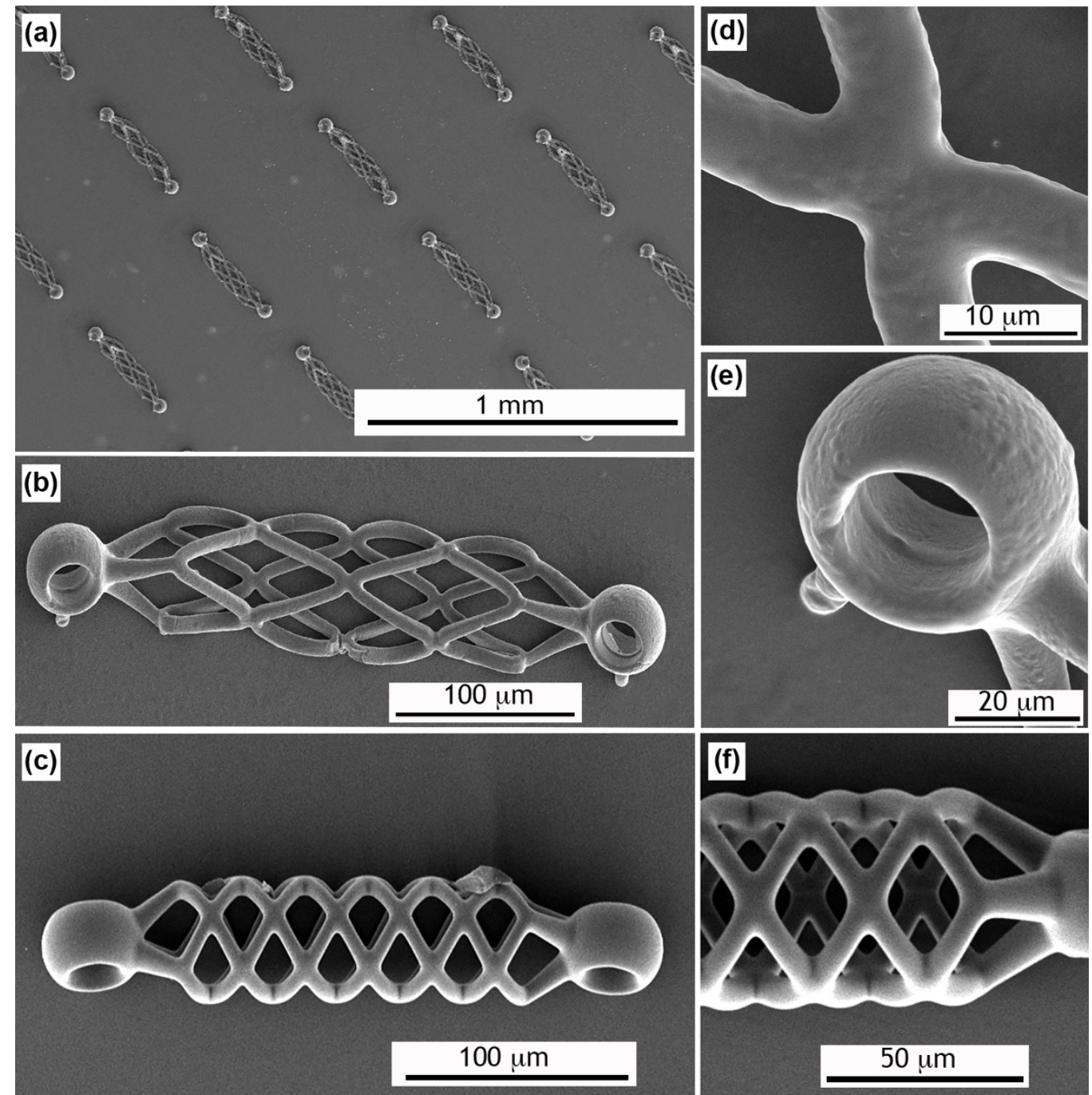

Figure 4 SEM images of the indirect 4D printed microstructures: array of stent-like microstructures (a), acuteangle (b), and obtuse (c,) the microstent strut (d), the acute microstent hook (e), and magnification of the obtuse mocrostent (f).

Previous fabrications of 4D printed cardiovascular stents have had diameters of approximately $5 \mathrm{~mm}$ and minimum resolution features of $\sim 200 \mu \mathrm{m}{ }^{[50]}$. Our 4D printed microstructures have minimum features of $5 \mu \mathrm{m}$, which is 40 times smaller than any other structures reported to date. While the Young's modulus $(E)$ for the reported 4D printed stent is around $100 \mathrm{MPa}^{[50]}$, the $E$ of the our microstent is in the order of $1 \mathrm{GPa}$ (Figure 5), which is comparable with those of commercial polymeric medical stents ${ }^{[51]}$. Although NOA63 is widely used and has outstanding properties, including its easily tunable glass transition temperature $\left(\mathrm{T}_{\mathrm{g}}\right)$ through a simple thermal post-exposure curing ${ }^{[52]}$, the use of NOA63 as an SMP for 4D printing microstructures has not yet been documented. According to previous studies, the $\mathrm{T}_{\mathrm{g}}$ of 


\section{WILEY-VCH}

NOA63 polymerized at room temperature is $29.7{ }^{\circ} \mathrm{C}$. To achieve a $\mathrm{T}_{\mathrm{g}}$ close to body temperature, it should be enough to postcure the polymer for 1 hour at $50^{\circ} \mathrm{C}^{[52]}$. As we used a UV lamp with a power density of $2.7 \mathrm{~mW} / \mathrm{cm}^{2}$, which is roughly half of the power density $\left(4.8 \mathrm{~mW} / \mathrm{cm}^{2}\right)$ of the UV lamp used by DiOrio et al., we carried out a first curing of 2 hours. To check the feasibility of tuning the glass transition temperature, we have also performed a post curing at $50^{\circ} \mathrm{C}$ for 120 minutes.

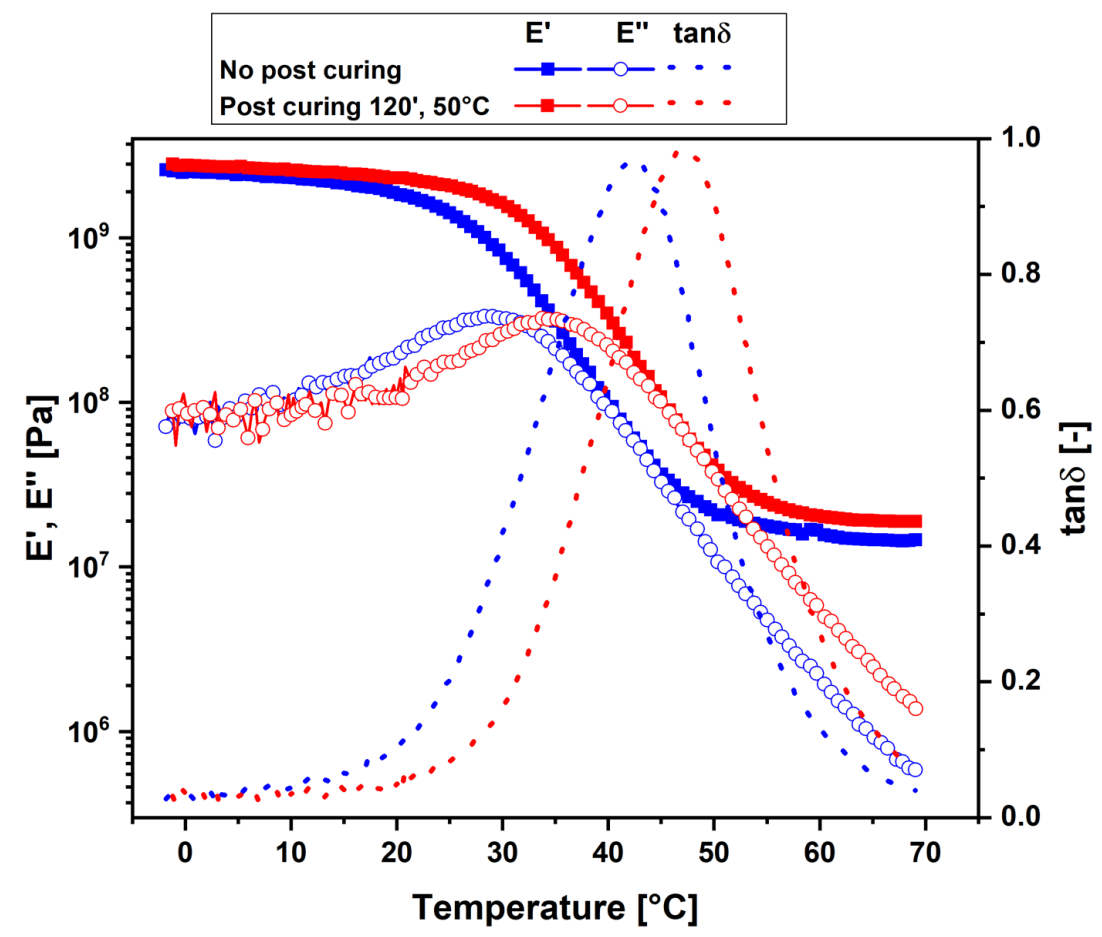

Figure 5 DMTA measurements on NOA63 films, UV-cured for 120 minutes at room-temperature without post curing (blue) and after post-curing for 120 minutes at $50{ }^{\circ} \mathrm{C}$ (red).

The Dynamic-Mechanics Thermal Analysis (DMTA) was selected to characterize the SMP, as it provides information on both the $\mathrm{T}_{\mathrm{g}}$, at which the shape recovery phenomenon occurs, and the entity of the modulus variation during the transition, which gives a good indication of the effectiveness of the shape recovery.

The results reported in Figure 5 confirm that the thermomechanical behavior of NOA63 can be tuned through UV crosslinking with a subsequent thermal treatment: in fact, the $\mathrm{T}_{\mathrm{g}}$, taken as the peak in the tan $\delta$ curve, increases from $42^{\circ} \mathrm{C}$ to $47^{\circ} \mathrm{C}$ if the post curing process is 


\section{WILEY-VCH}

applied. The conservative and dissipative components of modulus E' and E" respectively, also show an increase in the post-cured sample, confirming its higher degree of crosslinking. The DMTA measurements show that it is not necessary to postcure the samples to set the $\mathrm{T}_{\mathrm{g}}$ of the microstents to a temperature close to body temperature. In order to assess the shape memory behavior of the 4D printed microstructures, we first had to isolate individual microstents. To do this, we glued a $15 \mu \mathrm{m}$ tungsten wire to a needle and used a micromanipulator to thread it through one of the hooks (Figure 6a), picking up the other hook with a $22 \mu \mathrm{m}$ tungsten tip (Figure 6b). The microstent was stretched after heating it to above its $\mathrm{T}_{\mathrm{g}}$ (Figure 6c) using a heat gun (Steinel HG2320E), and the temperature close to the stent was measured using a temperature sensor (Sensirion STS21). Cold water was poured on the stent after lengthening. The microstent remained crimped in this frozen shape even after one of the tungsten tips was removed (Figure 6d). 


\section{WILEY-VCH}
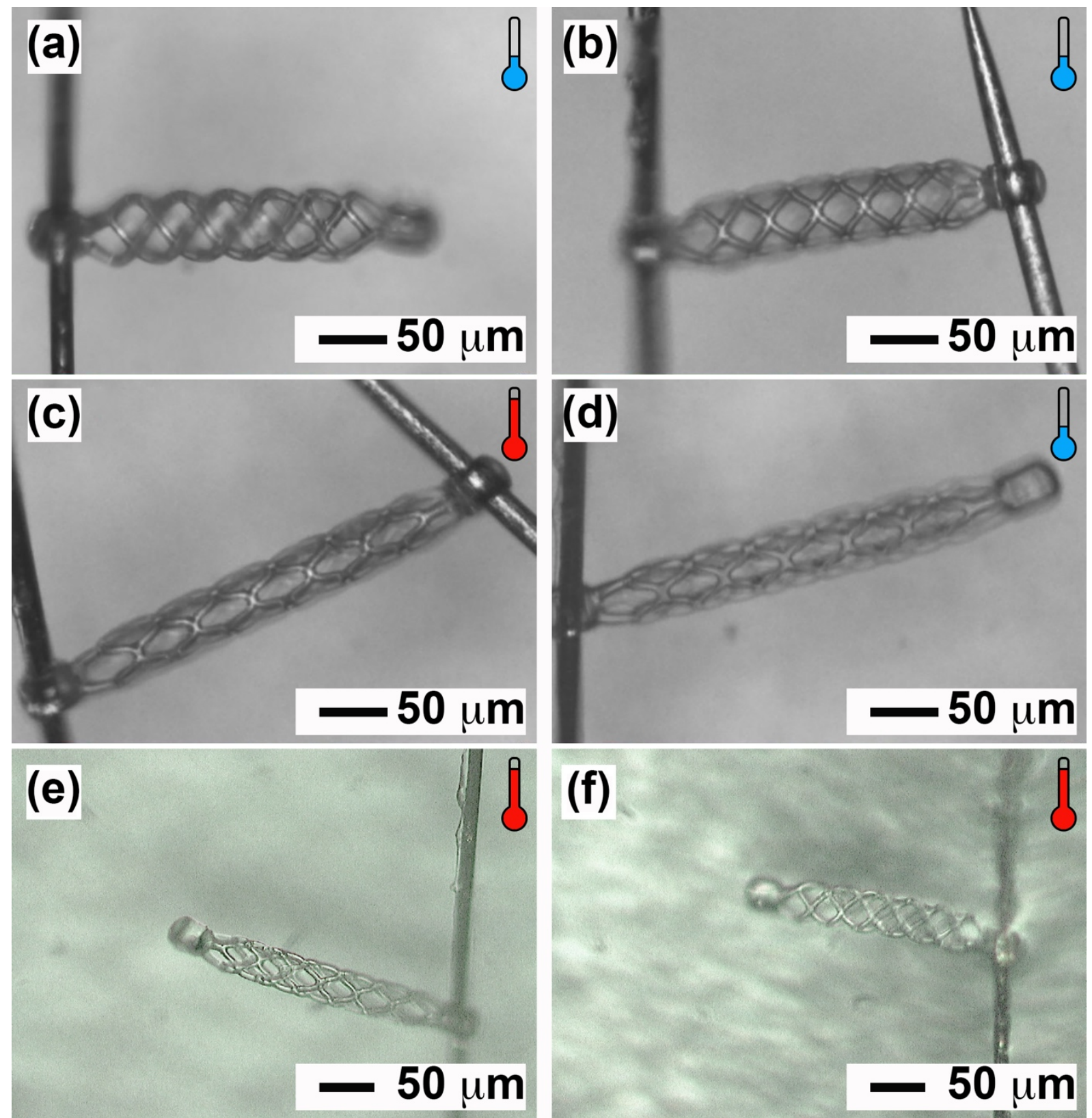

Figure 6 Optical microscope images showing the microstent at room temperature in its expanded state (a), before stretching (b), crimped above its Tg (c) and "frozen" in its new shape (d). The microstent was heated above its $\operatorname{Tg}(\mathrm{e})$ until it recovered its original shape (f) (The thermometer indicates a temperature below or above the $\mathrm{T}_{\mathrm{g}}$ in blue or red respectively).

To assess the shape memory properties, the microstent was first transferred to a Petri dish containing cold water, which was heated to $40{ }^{\circ} \mathrm{C}$ on a hotplate, to mimic the water-based environment of the body. The microstent recovered its original shape. We took one frame of the video (video S1) at the beginning of the experiment (Figure 6e), showing the microstent in its crimped shape as it is starting to recover its original shape. We took a second frame after approximately 3 minutes, when the shape recovery phenomenon was complete (Figure 6f). 


\section{WILEY-VCH}

The recovery ratio, $\mathrm{R}_{\mathrm{r}}=\left(\mathrm{L}_{\text {prog }}-\mathrm{L}_{\text {end }}\right) /\left(\mathrm{L}_{\text {prog }}-\mathrm{L}_{0}\right)$, where $\mathrm{L}_{0}, \mathrm{~L}_{\text {prog }}$ and $\mathrm{L}_{\text {end }}$ are the stent lengths before programming the temporary shape, after programming, and at the end of recovery respectively, was approximately 0.7 .

In conclusion, we have demonstrated an indirect 3D printing strategy to fabricate soft robotic structures at the microscale. We obtained 3D helical-shaped microswimmers made from pure gelatin doped with MNPs, without having to functionalize gelatin with photoreactive compounds. We also fabricated 4D stent-like microstructures with minimum features of $5 \mu \mathrm{m}$, which are 40 times smaller than those reported to date.

This work overcomes the technical limitations associated with current 3D printing techniques, paving the way for the fabrication of $3 \mathrm{D}$ and $4 \mathrm{D}$ printed soft microrobots for future medical devices. We expect this work to be of the utmost importance in pioneering the development of new soft microrobots that can tackle still unsolved medical issues.

\section{Experimental section}

Materials: NOA63 (Norland Products, Inc.) was purchased from APM Technica AG. Gelatin from porcine skin, superparamagnetic nanoparticles $\left(\mathrm{Fe}_{3} \mathrm{O}_{4}, 20 \mathrm{~nm}\right.$ average diameter) and all the solvents used throughout the experiments were purchased from Sigma Aldrich.

The positive-tone photoresist AZ IPS-6050 and the relative developer AZ 826MIF were provided by MicroChemicals GmbH.

\section{Experimental set-ups:}

The 3D shape of the sacrificial templates was prepared by spin-coating $82 \mu \mathrm{m}$ of AZ IPS6050 for $18 \mathrm{~s}$, followed by a soft-bake process at $125^{\circ} \mathrm{C}$ for 15 minutes. The $3 \mathrm{D}$ shapes were defined using a commercial DLW system (Nanoscribe, GmbH), with 63X objective (NA 1.4), used in DiLL-mode and oil immersion mode respectively, to write the sacrificial templates for micro helices and stent-like microstructures. The Galvo scanning mode was used with the 


\section{WILEY-VCH}

scan speed set to $5000 \mu \mathrm{m} / \mathrm{s}$. After a post-exposure bake at $100{ }^{\circ} \mathrm{C}$ for $100 \mathrm{~seconds}$, the substrate was developed for 20 minutes in AZ826MIF, then rinsed in water and dried with a nitrogen gun, to acquire the desired template.

The thermomechanical behavior of the cured material was investigated using DynamicMechanics Thermal Analysis (DMTA), performed on a TA RSA III dynamical mechanical analyzer. Tests were performed in tension, by applying a dynamic strain with an amplitude of $0.01 \%$ at a frequency of $1 \mathrm{~Hz}$ on a $0.26 \mathrm{~mm}$ thick prismatic sample. The temperature was raised from $0^{\circ} \mathrm{C}$ to $70^{\circ} \mathrm{C}$ at $1^{\circ} \mathrm{C} / \mathrm{min}$.

The magnetic properties of gelatin films were characterized using a Vibrating Sample Magnetometer (VSM), MicroSense EZ9.

MiniMag, an electromagnetic system for 5-DOF wireless micromanipulation, was used for the swimming tests. The swimming tests were carried out in silicon oil $(5 \mathrm{cSt})$ under a rotating magnetic field of $20 \mathrm{mT}$ with variable frequencies.

A micromanipulator system (Signatone) and tungsten microcroprobes (T-4-22) were used during the manipulation of the stent-like microstructures.

\section{Conflict of Interest}

The authors declare no conflict of interest.

\section{Acknowledgements}

C. D. M. acknowledges the Marie Skłodowska-Curie Individual Fellowships (microMAGNETOFLUIDICS, No. 702128).

Received: ((will be filled in by the editorial staff))

Revised: ((will be filled in by the editorial staff)) Published online: ((will be filled in by the editorial staff)) 
WILEY-VCH 


\section{WILEY-VCH}

ToC: A versatile indirect 3D and 4D printing technique presented to fabricate softmicrostructures. 3D gelatin helices filled with magnetic nanoparticles and 4D stent-like microstructures with shape-memory properties, 40 times smaller than reported to date, were easily manufactured.

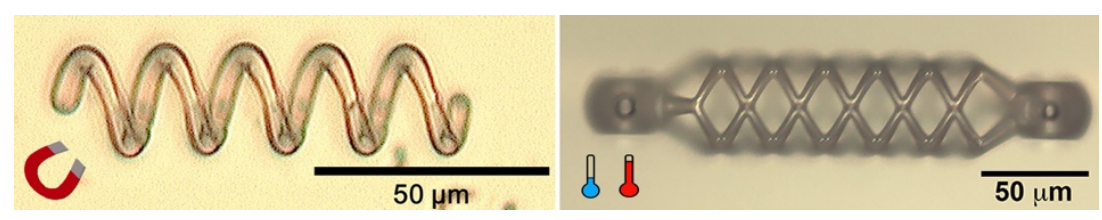




\section{WILEY-VCH}

\section{References}

[1] R. Mhanna, F. Qiu, L. Zhang, Y. Ding, K. Sugihara, M. Zenobi-Wong, B. J. Nelson, Small 2014, 10, 1953.

[2] S. Kim, F. Qiu, S. Kim, A. Ghanbari, C. Moon, L. Zhang, B. J. Nelson, H. Choi, Adv. Mater. 2013, 25, 5863.

[3] B. J. Nelson, I. K. Kaliakatsos, J. J. Abbott, Annu. Rev. Biomed. Eng. 2010, 12, 55.

[4] J. J. Abbott, K. E. Peyer, L. X. Dong, B. J. Nelson, Springer Tracts Adv. Robot. 2010, 66, 157.

[5] F. Qiu, L. Zhang, S. Tottori, K. Marquardt, K. Krawczyk, A. Franco-Obregón, B. J. Nelson, Mater. Today 2012, 15, 463.

[6] H.-W. Huang, M. S. Sakar, A. J. Petruska, S. Pane, B. J. Nelson, Nat Commun 2016, 7, 12263.

[7] H.-W. Huang, F. E. Uslu, P. Katsamba, E. Lauga, M. S. Sakar, B. J. Nelson, Sci. Adv. 2019, 5, 1 .

[8] S. Fusco, M. S. Sakar, S. Kennedy, C. Peters, R. Bottani, F. Starsich, A. Mao, G. A. Sotiriou, S. Pané, S. E. Pratsinis, D. Mooney, B. J. Nelson, Adv. Mater. 2014, $26,952$.

[9] C. Peters, M. Hoop, S. Pané, B. J. Nelson, C. Hierold, Adv. Mater. 2016, 28, 533.

[10] X. Wang, X.-H. Qin, C. Hu, A. Terzopoulou, X.-Z. Chen, T.-Y. Huang, K. ManiuraWeber, S. Pane, B. J. Nelson, Adv. Funct. Mater. 2018, 28, DOI 10.1002/adfm.201804107.

[11] T. Y. Huang, M. S. Sakar, A. Mao, A. J. Petruska, F. Qiu, X. B. Chen, S. Kennedy, D. Mooney, B. J. Nelson, Adv. Mater. 2015, 27, 6644.

[12] D. Walker, B. T. Käsdorf, H. H. Jeong, O. Lieleg, P. Fischer, Sci. Adv. 2015, 1, e1500501.

[13] F. Qiu, S. Fujita, R. Mhanna, L. Zhang, B. R. Simona, B. J. Nelson, Adv. Funct. Mater. 2015, 25, 1666.

[14] A. Vikram Singh, M. Sitti, Curr. Pharm. Des. 2016, 22, 1418.

[15] A. Servant, F. Qiu, M. Mazza, K. Kostarelos, B. J. Nelson, Adv. Mater. 2015, 27, 2981.

[16] X. Yan, Q. Zhou, M. Vincent, Y. Deng, J. Yu, J. Xu, T. Xu, T. Tang, L. Bian, Y.-X. J. Wang, K. Kostarelos, L. Zhang, Sci. Robot. 2017, 2, eaaq1155.

[17] W. Gao, R. Dong, S. Thamphiwatana, J. Li, W. Gao, L. Zhang, J. Wang, ACS Nano 2015, 9, 117.

[18] G. M. Whitesides, Angew. Chemie Int. Ed. 2018, 57, 4258.

[19] J. Rogers, Y. Huang, O. G. Schmidt, D. H. Gracias, MRS Bull. 2016, 41, 123.

[20] D. Rus, M. T. Tolley, Nat. Rev. Mater. 2018, 3, 101.

[21] J. Li, X. Li, T. Luo, R. Wang, C. Liu, S. Chen, D. Li, J. Yue, S. Cheng, D. Sun, Sci. Robot. 2018, DOI 10.1126/scirobotics.aat 8829.

[22] Y. Lin, J. Xu, Adv. Opt. Mater. 2018, DOI 10.1002/adom.201701359.

[23] X. Z. Chen, M. Hoop, F. Mushtaq, E. Siringil, C. Hu, B. J. Nelson, S. Pané, Appl. Mater. Today 2017, DOI 10.1016/j.apmt.2017.04.006.

[24] T. Qiu, T. C. Lee, A. G. Mark, K. I. Morozov, R. Münster, O. Mierka, S. Turek, A. M. Leshansky, P. Fischer, Nat. Commun. 2014, 5, DOI 10.1038/ncomms6119.

[25] W. Zhu, J. Li, Y. J. Leong, I. Rozen, X. Qu, R. Dong, Z. Wu, W. Gao, P. H. Chung, J. Wang, S. Chen, Adv. Mater. 2015, 27, 4411.

[26] T. J. Wallin, J. Pikul, R. F. Shepherd, Nat. Rev. Mater. 2018, DOI 10.1038/s41578018-0002-2.

[27] K. S. Lee, R. H. Kim, D. Y. Yang, S. H. Park, Prog. Polym. Sci. 2008, DOI 10.1016/j.progpolymsci.2008.01.001. 


\section{WILEY-VCH}

[28] J. K. Gansel, M. Thiel, M. S. Rill, M. Decker, K. Bade, V. Saile, G. Von Freymann, S. Linden, M. Wegener, Science (80-. ). 2009, 325, 1513.

[29] M. A. Zeeshan, R. Grisch, E. Pellicer, K. M. Sivaraman, K. E. Peyer, J. Sort, B. Özkale, M. S. Sakar, B. J. Nelson, S. Pané, Small 2014, 10, 1284.

[30] C. C. J. Alcântara, S. Kim, S. Lee, B. Jang, P. Thakolkaran, J.-Y. Kim, H. Choi, B. J. Nelson, S. Pané, Small 2019, 0, 1805006.

[31] X. Wang, Q. Ao, X. Tian, J. Fan, H. Tong, W. Hou, S. Bai, Polymers (Basel). 2017, 9, DOI 10.3390/polym9090401.

[32] S. Derakhshanfar, R. Mbeleck, K. Xu, X. Zhang, W. Zhong, M. Xing, Bioact. Mater. 2018, 3, 144.

[33] L. Zhang, J. J. Abbott, L. Dong, B. E. Kratochvil, D. Bell, B. J. Nelson, Appl. Phys. Lett. 2009, 94, 64107.

[34] L. Zhang, K. E. Peyer, B. J. Nelson, Lab Chip 2010, 10, 2203.

[35] J. Torgersen, X. H. Qin, Z. Li, A. Ovsianikov, R. Liska, J. Stampfl, Adv. Funct. Mater. 2013, DOI 10.1002/adfm.201203880.

[36] C. de Marco, S. Pané, B. J. Nelson, Sci. Robot. 2018, DOI 10.1126/scirobotics.aau0449.

[37] S. Tibbits, Archit. Des. 2014, 84, 116.

[38] Y. C. Li, Y. S. Zhang, A. Akpek, S. R. Shin, A. Khademhosseini, Biofabrication 2017, 9, DOI 10.1088/1758-5090/9/1/012001.

[39] Z. Ding, C. Yuan, X. Peng, T. Wang, H. J. Qi, M. L. Dunn, Sci. Adv. 2017, 3, e1602890.

[40] Q. Ge, H. J. Qi, M. L. Dunn, Appl. Phys. Lett. 2013, 103, DOI 10.1063/1.4819837.

[41] H. Wei, Q. Zhang, Y. Yao, L. Liu, Y. Liu, J. Leng, ACS Appl. Mater. Interfaces 2017, DOI 10.1021/acsami.6b12824.

[42] Y. Kim, H. Yuk, R. Zhao, S. A. Chester, X. Zhao, Nature 2018, DOI 10.1038/s41586018-0185-0.

[43] X. Kuang, K. Chen, C. K. Dunn, J. Wu, V. C. F. Li, H. J. Qi, ACS Appl. Mater. Interfaces 2018, DOI 10.1021/acsami.7b18265.

[44] Q. Mu, C. K. Dunn, L. Wang, M. L. Dunn, H. J. Qi, T. Wang, Smart Mater. Struct. 2017, DOI 10.1088/1361-665X/aa5cca.

[45] A. S. Wu, W. Small, T. M. Bryson, E. Cheng, T. R. Metz, S. E. Schulze, E. B. Duoss, T. S. Wilson, Sci. Rep. 2017, DOI 10.1038/s41598-017-04663-z.

[46] K. Chen, X. Kuang, V. Li, G. Kang, H. J. Qi, Soft Matter 2018, DOI $10.1039 / \mathrm{c} 7 \mathrm{sm} 02362 \mathrm{f}$.

[47] S. Maruo, O. Nakamura, S. Kawata, Opt. Lett. 1997, DOI 10.1364/OL.22.000132.

[48] E. P. Dupont, R. Luisier, M. A. M. Gijs, Microelectron. Eng. 2010, 87, 1253.

[49] C. Liberale, G. Cojoc, P. Candeloro, G. Das, F. Gentile, F. De Angelis, E. Di Fabrizio, IEEE Photonics Technol. Lett. 2010, DOI 10.1109/LPT.2010.2040986.

[50] Q. Ge, A. H. Sakhaei, H. Lee, C. K. Dunn, N. X. Fang, M. L. Dunn, Sci. Rep. 2016, 6, DOI 10.1038/srep31110.

[51] D. Y. Kwon, J. Il Kim, D. Y. Kim, H. J. Kang, B. Lee, K. W. Lee, M. Suk, K. 1\#, J. Biomed. Sci. Eng. 2012, 5, 208.

[52] A. M. DiOrio, X. Luo, K. M. Lee, P. T. Mather, Soft Matter 2011, 7, 68. 